\title{
Distinct Domains within PSD-95 Mediate Synaptic Incorporation, Stabilization, and Activity-Dependent Trafficking
}

\author{
James F. Sturgill, ${ }^{\star}$ Pascal Steiner, ${ }^{\star}$ Brian L. Czervionke, and Bernardo L. Sabatini \\ Howard Hughes Medical Institute, Department of Neurobiology, Harvard Medical School, Boston, Massachusetts 02115
}

\begin{abstract}
The postsynaptic density (PSD) consists of a lattice-like array of interacting proteins that organizes and stabilizes receptors, ion channels, structural, and signaling proteins necessary for synaptic function. To study the stabilization of proteins within this structure and the contribution of these proteins to the integrity of the PSD, we tagged synaptic proteins with PAGFP (photoactivatable green fluorescent protein) and used combined two-photon laser-scanning microscopy and two-photon laser photoactivation to measure their rate of turnover in individual spines of rat CA1 pyramidal neurons. We find that PSD-95 is highly stable within the spine, more so than other PSD-associated proteins such as CaMKII $\alpha$, CaMKII $\beta$, GluR2, and Stargazin. Analysis of a series of PSD-95 mutants revealed that distinct domains stabilize PSD-95 within the PSD and contribute to PSD formation. Stabilization of PSD-95 within the PSD requires N-terminal palmitoylation and protein interactions mediated by the first and second PDZ domains, whereas formation of a stable lattice of PSD-95 molecules within the PSD additionally requires the C-terminal SH3 domain. Furthermore, in a PDZ domain 1 and 2 dependent manner, activation of NMDA receptors with a chemical long-term depression protocol rapidly destabilizes PSD-95 and causes a subset of the PSD-95 molecules previously anchored in the spine to be released. Thus, through the analysis of rates of exchange of synaptic PSD-95, we determine separate domains of PSD-95 that play specific roles in establishing a stable postsynaptic lattice, in allowing proteins to enter this lattice, and in reorganizing this structure in response to plasticity-inducing stimuli.
\end{abstract}

\section{Introduction}

Structural and molecular rearrangements of synapses are thought to underlie the expression of many forms of synaptic plasticity. At excitatory synapses, these rearrangements occur within dendritic spines at the postsynaptic density (PSD), an electron-dense thickening at the membrane that houses proteins that respond to and regulate synaptic transmission (Scannevin and Huganir, 2000; Inoue and Okabe, 2003; Kim and Sheng, 2004). That the structure of the PSD is preserved during cell-fractionation and stringent detergent washes indicates that it is a highly stable structure comprised of tightly anchored proteins. Yet, during the induction of activity-dependent forms of plasticity, synaptic proteins such as AMPA- and NMDA-type glutamate receptors (AMPARs and NMDARs, respectively), as well as kinases (CaMKII), phosphatases, and regulators of the actin cytoskeleton must be added to or released from the PSD (Shen and Meyer, 1999; Okabe et al., 2001; Malinow and Malenka, 2002; Ehlers, 2003;

Received April 17, 2009; revised Aug. 31, 2009; accepted Sept. 1, 2009.

This work was funded by grants from the Dana and National Alliance for Research on Schizophrenia and Depression Foundations, National Institute of Neurological Disorders and Stroke (Grant NS052707), and the Swiss National Science Foundation (Grant PA00A-113192/1; to P.S.).We thank the Sabatini laboratory for helpful comments and discussions.

*J.F.S. and P.S. contributed equally to this work.

Correspondence should be addressed to Bernardo L. Sabatini, Howard Hughes Medical Institute, Department of Neurobiology, Harvard Medical School, 220 Longwood Avenue, Boston, MA 02115. E-mail: bsabatini@hms. harvard.edu.

DOI:10.1523/JNEUROSCI.1841-09.2009

Copyright $\odot 2009$ Society for Neuroscience $\quad 0270-6474 / 09 / 2912845-10 \$ 15.00 / 0$
Otmakhov et al., 2004; Gray et al., 2006; Smith et al., 2006; Bellone and Nicoll, 2007).

The protein-protein interactions that endow the PSD with its basal structural stability as well as those that are regulated to allow transient plasticity are unclear. While the exhaustive set of potential interactions is enormous, a subset of abundant proteins such as CaMKII $\alpha$, CaMKII $\beta$, SynGAP, and PSD-95/SAP90 may form interactions that play a dominant role in determining PSD stability (Sheng and Hoogenraad, 2007). Electron microscopic analysis suggest that some of these proteins form an organized lattice that may underlie the structural integrity of the PSD (Chen et al., 2008).

In particular, PSD-95, a member of the membrane-associated guanylate kinase (MAGUK) family of scaffolding proteins, is believed to regulate basal stability as well as activitydependent structural plasticity of the PSD. Previous studies have shown that PSD-95 is highly enriched at synapses and have identified multiple protein domains and interactions that mediate PSD-95 trafficking to the postsynaptic terminal (Craven et al., 1999). Furthermore, PSD-95 is highly stable at the synapse as demonstrated by fluorescence photobleaching and photoactivation studies, consistent with a role in forming the PSD (Gray et al., 2006; Kuriu et al., 2006; Yoshii and ConstantinePaton, 2007; Blanpied et al., 2008; Steiner et al., 2008; Xu et al., 2008).

To identify proteins that might be necessary for PSD stability, we consider a representative set of abundant and/or relevant synaptic proteins including PSD-95. We express versions of these 
proteins tagged with photoactivatable green fluorescent protein (PAGFP) in CA1 hippocampal pyramidal neurons and compare their stabilities within dendritic spines. Measurement of fluorescence decay rates after photoactivation reveal that PSD-95 is more stable than the other proteins examined, consistent with a core role in stabilizing the PSD. Examination of PSD-95 mutants identifies domains that allow PSD-95 to form a stable protein network within the PSD, to integrate into such a preexisting network, and to translocate out of the spine in response to chemical induction of NMDAR-dependent long-term depression (LTD). Therefore, although PSD-95 stabilizes itself and likely determines the stability of other proteins, destabilization of PSD-95 may initiate the exchange and turnover of synaptic proteins necessary for the induction of LTD.

\section{Materials and Methods}

Hippocampal slice cultures and transfection. Hippocampal slice cultures were prepared from postnatal day 5-7 Sprague Dawley rats as described previously (Stoppini et al., 1991). DNA constructs were transfected biolistically into slice cultures after 2-4 din vitro (DIV) using a Helios Gene Gun (Biorad). Unless otherwise noted, bullets were prepared from $40 \mu \mathrm{g}$ of each of PAGFP-tagged constructs and dsRed-T1 using $12.5 \mathrm{mg}$ of 1.6 $\mu \mathrm{m}$ gold particles. Slices were maintained as interface cultures on MilliCell Culture Plate Inserts (Millipore) until imaging at 10-14 DIV. For Figure $3 F$, slices were imaged $10-11 \mathrm{~d}$ posttransfection (DPT) to allow for effective knock-down of endogenous NR1 (Alvarez et al., 2007). For Figure $4 F$, slices were imaged 2-3 DPT to compensate for differences in expression levels seen with standard and shPSD-95 constructs.

DNA constructs. PSD-95-PAGFP, $\triangle$ PDZ1/2 PSD-95-PAGFP, $\triangle$ PDZ3/ SH3/GK PSD-95-PAGFP, $\Delta$ SH3/GK PSD-95-PAGFP, sh95 + PSD-95PAGFP $\Delta$ GK, sh95 + PSD-95-PAGFP $\Delta$ SH3/GK and shNR1 constructs were generated as described (Craven et al., 1999; Koh et al., 1999; Alvarez et al., 2007; Steiner et al., 2008; Xu et al., 2008). The following DNA constructs were obtained as gifts: pPAGFP-C1 (J. Lippincott-Schwartz, National Institute of Child Health and Human Development, Bethesda, MD), pCFP-GluR2-GW1 (H. Hirling, Ecole Polytechnique Fédérale de Lausanne, Lausanne, Switzerland), pEGFP-CaMKII $\alpha$ and pEGFPCaMKII $\beta$ (T. Meyer, Stanford University, Palo Alto, CA), and pStargazin-EGFP-GW1 (R.A. Nicoll, University of California, San Francisco, San Francisco, CA). PAGFP-GluR2 and Stargazin-PAGFP were generated by ligation of PCR-amplified PAGFP in frame to replace the existing XFP using the sites XbaI and BglII/NotI, respectively. CaMKII $\alpha$ PAGFP and CaMKII $\beta$-PAGFP were generated by amplifying the cDNAs of CaMKII $\alpha$ and CaMKII $\beta$ and subcloning them into the PAGFP-C1 vector at the sites EcoRI/KpnI. The non-palmitoylated mutant of PSD-95, PSD-95 C3,5S-PAGFP was generated by mutating the cysteine residues at position 3 and 5 to serine residues via standard PCR methods (Craven et al., 1999).

Imaging and pharmacological treatments. Experiments were conducted at room temperature $\left(20-22^{\circ} \mathrm{C}\right)$ in artificial CSF (ACSF, in mM: 127 $\mathrm{NaCl}, 25 \mathrm{NaHCO}_{3}, 1.25 \mathrm{NaHPO}_{4}, 2.5 \mathrm{KCl}, 1 \mathrm{MgCl}_{2}, 25$ D-glucose, 2.5 $\mathrm{CaCl}_{2}$ ) saturated with $95 \% \mathrm{O}_{2}$ and $5 \% \mathrm{CO}_{2}$. NMDA stimulation consisted of 20-40 $\mu \mathrm{M}$ NMDA in ACSF over 3-4 min. When indicated, hippocampal slices were preincubated with $1 \mu \mathrm{M}$ TTX for $1 \mathrm{~h}, 20 \mu \mathrm{M}$ CPP for $30 \mathrm{~min}, 1 \mu \mathrm{M}$ Calyculin A for $30 \mathrm{~min}$, or $1 \mu \mathrm{M}$ FK-506 for $1 \mathrm{~h}$ and imaged in the continued presence of these drugs. Transfected CA1 pyramidal neurons were visualized and selected based upon characteristic pyramidal morphology. Spiny regions of secondary or tertiary apical dendrites were imaged at high magnification using a custom two-photon laser scanning and two-photon laser photoactivation microscope (Steiner et al., 2008). For photoactivation and imaging, the laser wavelengths and powers measured at the back aperture of the objective were $730 \mathrm{~nm} / \sim 35 \mathrm{~mW}$ and $920 \mathrm{~nm} / 68-75 \mathrm{~mW}$, respectively. Image stacks of spiny dendrites were collected at a $\mathrm{z}$-spacing of $1 \mu \mathrm{m}$ and a frame resolution of $256 \times 256$ pixels. Photoactivation occurred in a user-selected region of interest (ROI) surrounding the spine in the z-slice containing the highest intensity dsRed fluorescence within the ROI as well as in the slices immediately above and below this slice. An image stack of the same dendritic segment was acquired $\sim 1$ min after photoactivation and then every $5 \mathrm{~min}$. A second photoactivation protocol was delivered to the same area $30 \mathrm{~min}$ after the first to detect changes in the size of the pool of PAGFP-tagged protein within the spine.

Data analysis. DsRed and PAGFP fluorescence was quantified using custom software written in Matlab (MathWorks). For each spine and at each time point, the user marked lines corresponding to a major axis along the length of the spine and a minor axis intersecting the major axis at the point of maximal dsRed intensity in the spine head. The maximal intensity of dsRed was used to define an area around the spine in which the fluorescence intensity remained $>30 \%$ of this maximal value. This area was designated as the spine head mask. In addition, the $30 \%$ fluorescence fall off point was calculated along the minor and major axis to define, respectively, the width and length of the spine head. Only information from the dsRed channel was used to calculate the spine head mask, spine head width, and spine length. The total green fluorescence within the spine head mask was calculated at each time point and expressed relative to the increase in green fluorescence within the spine head mask triggered by the initial photoactivating pulse. This proportional value is referred to as $F_{\mathrm{PAGFP}}$. The time of acquisition of the first image after the photoactivating pulse is referred to as $t=0 \mathrm{~min}$ and by definition $F_{\mathrm{PAGFP}}$ at this time is $100 \%$. In supplemental Figure 2, available at www.jneurosci.org as supplemental material, the spine head area refers to the number of pixels in the spine head mask. All analysis was performed on the raw fluorescence intensities recorded by the imaging data acquisition devices. Figures were prepared in Adobe Photoshop and Illustrator. For display purposes, a linear color scale was chosen for each PAGFPfusion protein and kept constant in each time series displaying neurons transfected with that fusion protein. Data are expressed as mean values \pm SEM and unpaired, two-tailed $t$ tests were used for all statistical comparisons.

Experimental design. To study the dynamics of spine proteins, we used two-photon laser-scanning microscopy (2PLSM) combined with twophoton laser photoactivation (2PLP) of PAGFP-tagged synaptic proteins including PSD-95 (Gray et al., 2006; Steiner et al., 2008). This approach allowed us to measure the turnover of PAGFP-tagged synaptic proteins at the level of individual dendritic spines. Previous studies have examined synaptic protein mobility using fluorescence recovery after photobleaching (FRAP) (Kuriu et al., 2006; Sharma et al., 2006; Tsuriel et al., 2006). In FRAP studies of trafficking of synaptic proteins in spines, fluorescence recovery of the mobile pool of protein is monitored and the size of the stable pool of protein is calculated from the unrecoverable fraction of initial fluorescence. Thus, stimulus-evoked changes in the size of the stable pool over time cannot be assayed. In addition, because of the resistance of GFP and many of its derivatives to photobleaching and the inherently poor signal-to-noise of FRAP for monitoring nondiffusing proteins, photodamage to areas of interest is more likely (Patterson and Lippincott-Schwartz, 2004).

Overexpression of PSD-95 in dissociated cultured neurons or hippocampal slices enhanced basal synaptic transmission and spine size (El-Husseini et al., 2000a; Stein et al., 2003; Choe and Ehrlich, 2006). Yet other studies using PSD-95 overexpression did not reveal effects on synaptic strength or morphology (Gray et al., 2006; Kelsch et al., 2008). Such variability may relate to heterogeneity in PSD-95 expression levels. We have previously shown that the responses of normal sized spines in PSD-95 expressing neurons to two-photon glutamate uncaging were indistinguishable from control neurons and that these spines expressed both structural and functional plasticity in response to stimuli that induce long-term potentiation (Steiner et al., 2008).

In the majority of our experiments, PAGFP-tagged proteins are introduced in the presence of endogenous protein. Our initial goal was not to study the process of synapse formation but rather the molecular pathways that control the stability and numbers of tethered proteins within the spine. For example, we find that C3,5S PSD-95, which cannot be palmitoylated, cannot be stably incorporated into the postsynaptic terminal. If this mutant was expressed in the absence of endogenous PSD95, there would be no population of stable endogenous PSD-95 and lack of incorporation of depalmitoylated PSD-95 into this pool would be a trivial result. 


\section{A PSD95-PAGFP}
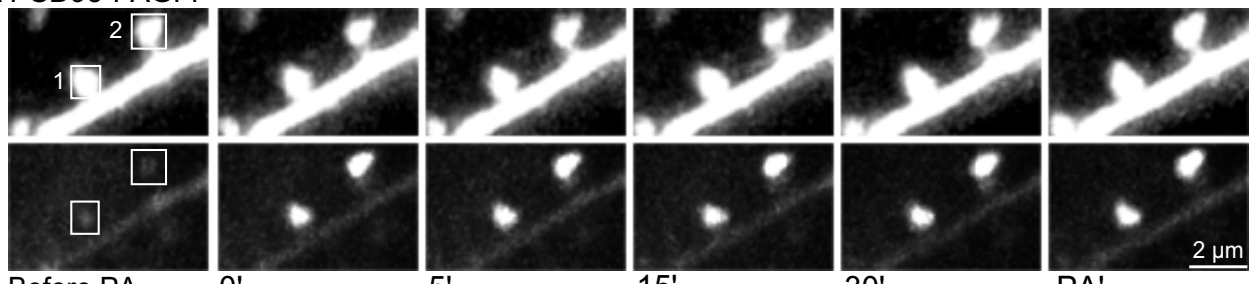

Before PA
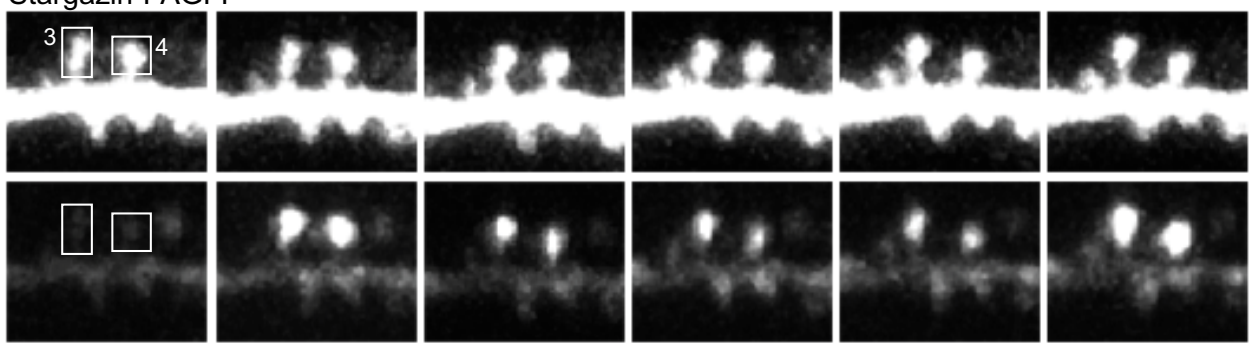

C CaMKIl $\alpha$-PAGFP
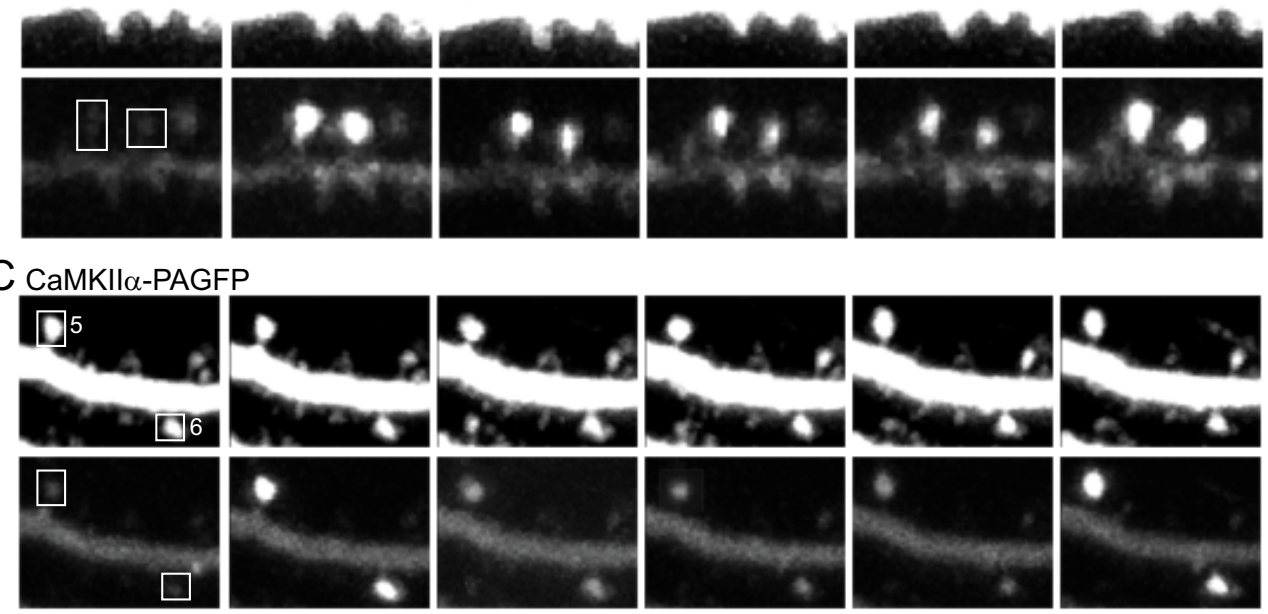

D
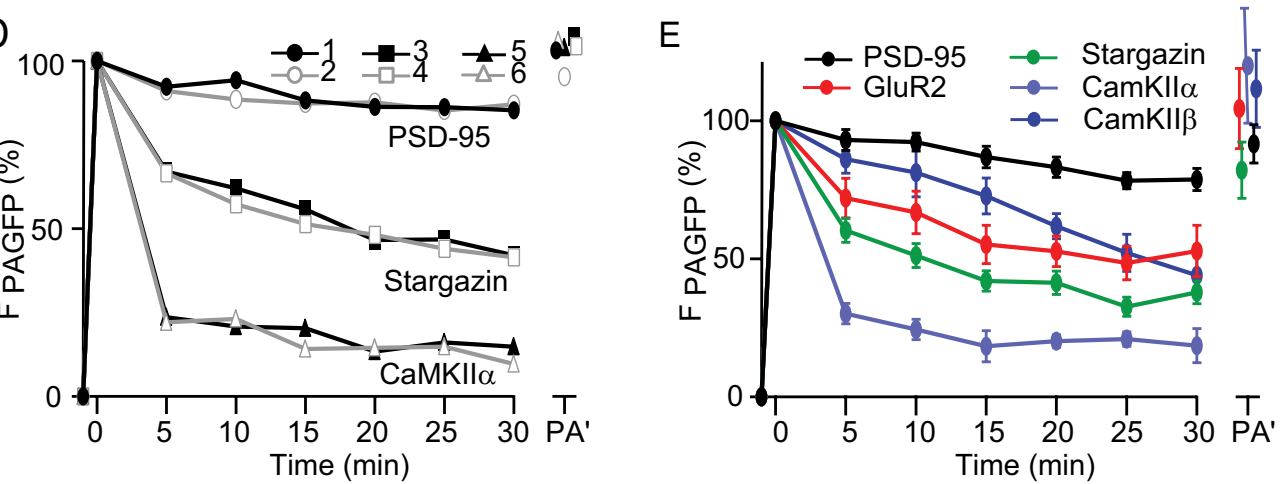

Figure 1. Differential stability of synaptic proteins revealed by time-lapse imaging of PAGFP-tagged proteins in individual dendritic spines. $A$, Representative images of a spiny region of apical dendrite of a CA1 pyramidal neuron expressing dsRed and PSD-95-PAGFP. Red fluorescence from dsRed (top) was used to monitor spine morphology while green fluorescence from PSD-95-PAGFP (bottom) was used to track the movement of PSD-95. PSD-95-PAGFP in two spines was photoactivated (time $t=0 \mathrm{~min}$ ) by two-photon excitation with $730 \mathrm{~nm}$ laser light in the indicated areas (spines 1 and 2). In this and all subsequent figures, the white boxes indicate the photoactivated areas. Fluorescence intensity was monitored with time-lapse imaging at 5 min intervals during 30 $\mathrm{min}$. At the end of the imaging period, the spines were exposed to a second photoactivating pulse (PA'). $\boldsymbol{B}, \boldsymbol{C}$, As in $\boldsymbol{A}$, for neurons expressing dsRed and either Stargazin-PAGFP ( $\boldsymbol{B}$, spines 3 and 4$)$ or CaMKII $\alpha$-PAGFP ( $\boldsymbol{C}$, spines 5 and 6). D, Quantification of green fluorescence in the spine head as a function of time for the spines indicated in $\boldsymbol{A}-\boldsymbol{C}$. $\boldsymbol{E}$, Average time courses of PSD-95-PAGFP, GluR2-PAGFP, Stargazin-PAGFP, CaMKII $\alpha$-PAGFP, and CaMKII $\beta$-PAGFP fluorescence in spines following photoactivation $(n=45 / 9,33 / 13,21 / 4,12 / 4$, and $9 / 2$ spines/cells, respectively), revealing distinct dynamics of each protein.

\section{Results}

Synaptic protein dynamics in individual spines

To monitor the dynamics of synaptic proteins at the level of individual dendritic spines, we tagged CaMKII $\alpha$, CaMKII $\beta$, GluR2, PSD-95, and Stargazin with PAGFP (Patterson and LippincottSchwartz, 2002) and expressed each fusion protein along with the red fluorescent protein dsRed-T1 (dsRed) in hippocampal neurons in rat organotypic slices. At 10-12 d DPT, transfected CA1 pyramidal neurons were selected for analysis and 2PLSM (920 $\mathrm{nm}$ excitation) was used to image neuronal morphology and identify dendritic spines from the primary and secondary branches of apical dendrites (Fig. 1). Before photoactivation, little green fluorescence from PAGFP-tagged proteins was detectable in spines and dendrites (Fig. 1A-C; supplemental Fig. 1, available at www.jneurosci.org as supplemental material). Brief pulses of illumination at $730 \mathrm{~nm}$ with a second laser triggered 2PLP of PAGFP and large increases in green fluorescence in the targeted spine heads (Fig. $1 A-C$; supplemental Fig. 1, available at www.jneurosci.org as supplemental material) (Bloodgood and Sabatini, 2005; Gray et al., 2006; Blanpied et al., 2008; Steiner et al., 2008). Since photoactivation of PAGFP reflects a covalent modification of the fluorophore, green fluorescence after photo- 
activation directly reports the distribution of tagged proteins that had been exposed to the photoactivating pulse (Patterson and Lippincott-Schwartz, 2002, 2004).

Time-lapse imaging revealed that the majority of the PSD-95 seen in the spine in the first image acquired after the photoactivating pulse (defined as $t=0$ and acquired $\sim 1 \mathrm{~min}$ after the $730 \mathrm{~nm}$ pulse) remained in the head $30 \mathrm{~min}$ later (Fig. $1 A)$, consistent with previous reports (Gray et al., 2006; Steiner et al., 2008). Soluble PAGFP is fully cleared from spines by diffusional exchange with the dendrite within seconds (Bloodgood and Sabatini, 2005; Gray et al., 2006). Therefore, this retained pool reflects protein that is anchored within the spine and unable to diffuse away from the site of photoactivation. Exchange of PAGFP-tagged proteins between this stable structure and a possible freely diffusing pool of PSD-95PAGFP was tested by delivery of a second photoactivating pulse after $30 \mathrm{~min}$ of imaging. This second pulse produced a modest increase in green fluorescence, indicating only minimal new incorporation of unactivated PSD-95-PAGFP. In addition, spine PAGFP fluorescence levels reached after the first and second photoactivation pulses were equal, indicating that photobleaching or run-down of PSD95-PAGFP levels within the spine had not occurred.

In control experiments, photoactivation of spines expressing PSD-95-PAGFP at a range of power levels caused a step increase in fluorescence that achieved a plateau which was stable over successive, closely spaced photoactivation pulses (supplemental Fig. S2 A, available at www.jneurosci.org as supplemental material). Furthermore, rapid, repetitive imaging of photoactivated spines at varying power levels revealed that only with higher imaging powers and more imaging time points than used in this study did significant photobleaching of PSD-95PAGFP occur (supplemental Fig. S2 B, available at www.jneurosci. org as supplemental material). Finally, spine morphology was stable during prolonged imaging, consistent with a lack of significant photodamage (supplemental Fig. 2C-E, available at www.jneurosci. org as supplemental material). Therefore, based on these controls, the rate of decay of PSD-95-PAGFP fluorescence reported in Figure 1 reflects the rate of exchange of PSD-95 molecules between the spine head and the dendrite.

Similar analyses of Stargazin, CaMKII $\alpha$, CaMKII $\beta$, and GluR2 PAGFP fusion proteins (Fig. $1 B, C$; supplemental Fig. 1, available at www.jneurosci.org as supplemental material) demonstrated that these proteins are less stable in the spine following photoactivation such that a significant fraction of the green fluorescence is lost within $30 \mathrm{~min}$ (see also Sharma et al., 2006; Lee et al., 2009). Furthermore, significant incorporation of unactivated PAGFP tagged proteins occurred during this time as a second photoactivation pulse triggered large increases in green fluorescence in the selected spines. To quantify the stability of each protein in the spine head, the green fluorescence within the spine head above basal levels (see Materials and Methods) was expressed as a frac- tion of the increase in spine head green fluorescence induced by the photoactivating pulse $\left(F_{\text {PAGFP }}\right)$ and reported as a function of time $\left(F_{\text {PAGFP }}(t)\right)$ (Fig. $\left.1 D\right)$. Although stabilities of different proteins in the spine head differ, the results for each tagged protein are largely consistent across spines such that PSD-95 is more stable than Stargazin and the highly motile CaMKII $\alpha$ (Fig. $1 D, E)$. Similar analysis of GluR2-PAGFP and CaMKII $\beta$-PAGFP revealed that these proteins have stabilities in the spine that are intermediate to that of PSD-95 and CaMKII $\alpha$ (Fig. $1 E$ ). These results demonstrate that, as expected for proteins associated with the PSD, populations of these proteins are incorporated into a stable structure within the spine and that this tethering increases their lifetime in the spine far beyond that of freely diffusing proteins.

Palmitoylation of PSD-95 is required for the incorporation and stabilization of PSD-95 within the postsynaptic density The highly stable nature of PSD-95 is consistent with its role as a core structural component of the PSD (Kennedy, 2000; Sheng, 2001; Valtschanoff and Weinberg, 2001; Petersen et al., 2003; Kim and Sheng, 2004). Electron microscopic (EM) studies indicate that PSD-95 resides deep within the PSD near the synaptic cleft where it engages in interactions believed to be crucial for the formation of the PSD (Valtschanoff and Weinberg, 2001; Petersen et al., 2003). These interactions are mediated by multiple, distinct protein domains and are believed to serve the dual functions of participating in the formation and organization of the PSD and of stabilizing PSD-95 within it. Previous studies have demonstrated that synaptic targeting and clustering of PSD-95 requires palmitoylation at cysteines 3 and 5 
A
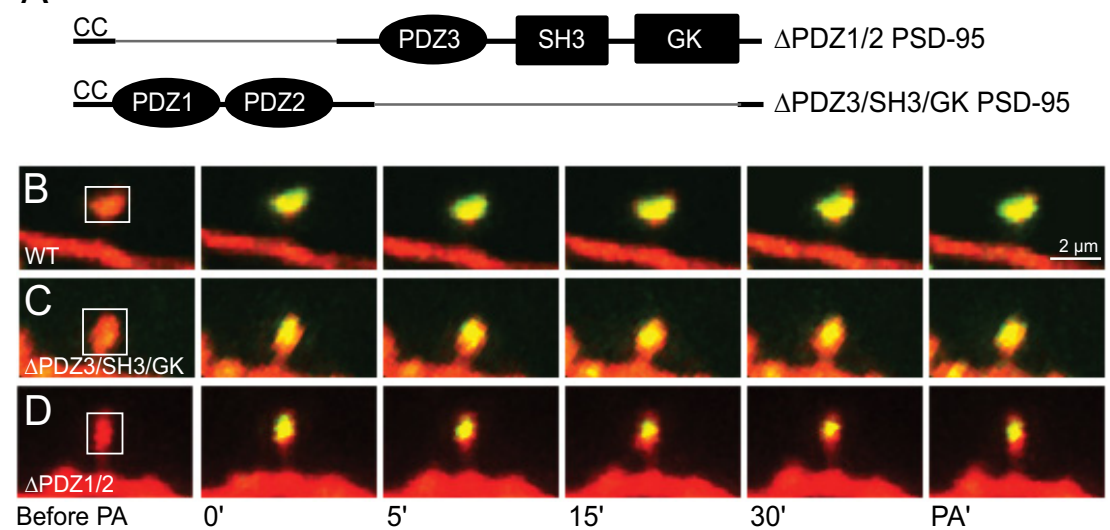

Before PA
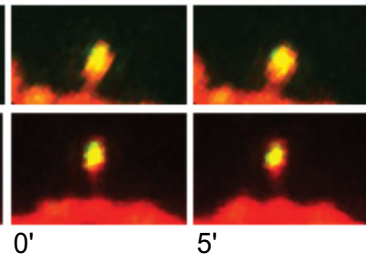

E

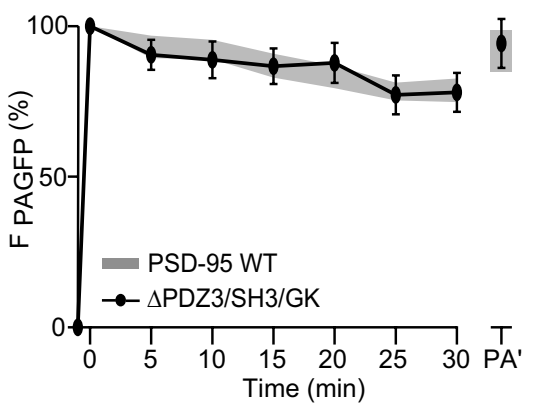

$\mathrm{F}$

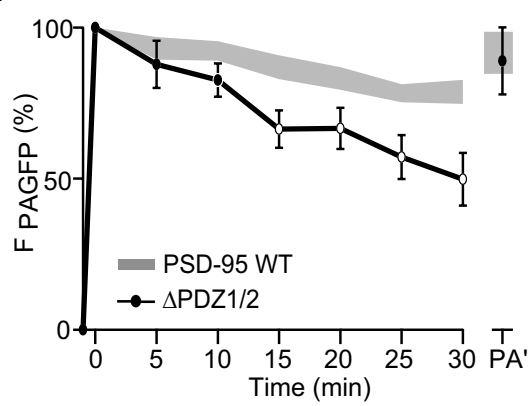

$\triangle P D Z 1 / 2-P A G F P+$ WT-untagged
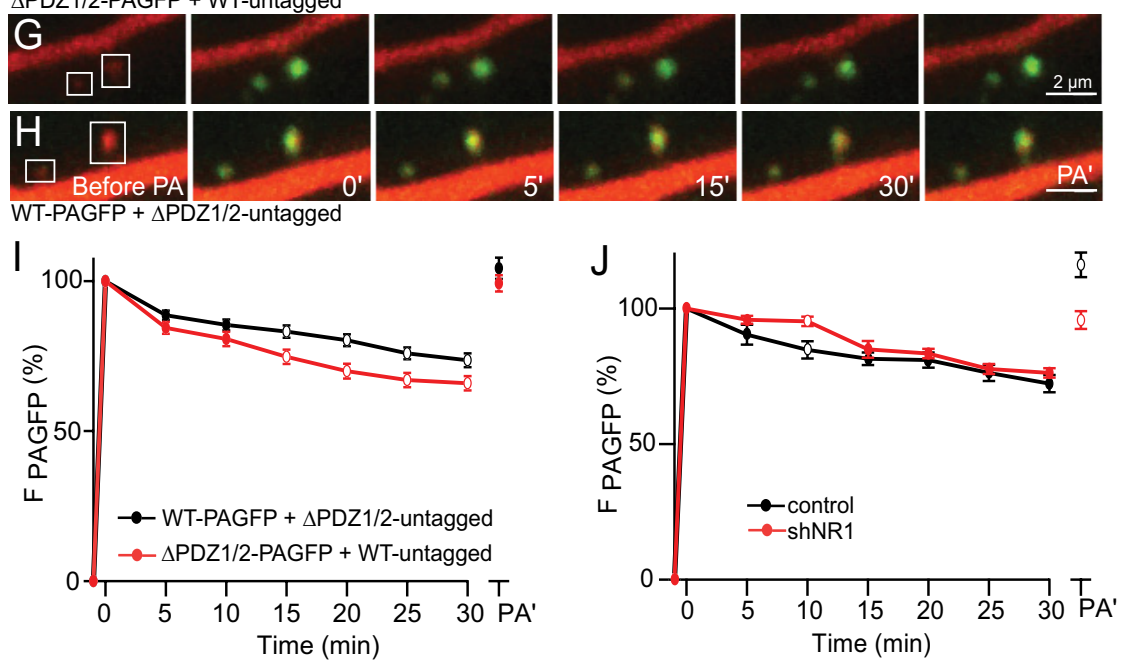

Figure 3. N-terminal domains of PSD-95 including PDZ1/2 are required for full stabilization of PAGFP-tagged PSD-95. $\boldsymbol{A}$, Schematic representations of $\mathrm{N}$-terminal $(\triangle \mathrm{PDZ1} / 2)$ and -terminal $(\triangle \mathrm{PDZ3} / \mathrm{SH} 3 / \mathrm{GK})$ mutants of PSD-95. $\boldsymbol{B}-\boldsymbol{D}$, Representative image series of photoactivated spines in neurons expressing either WT (B), $\Delta$ PDZ3/SH3/GK (C), or $\Delta$ PDZ1/2 (D) PSD-95-PAGFP together with dsRed. $\boldsymbol{E}, \boldsymbol{F}$, Average time courses of fluorescence for $\Delta$ PDZ3/SH3/GK (E, circles, $n=61 / 17$ spines/cells) or $\Delta$ PDZ1/2 ( $F$, circles, $n=37 / 17$ spines/cells) PSD-95-PAGFP compared to WT PSD-95-PAGFP. $\boldsymbol{G}, \boldsymbol{H}$, As in $\boldsymbol{B}-\boldsymbol{D}$ for neurons expressing either $\triangle$ PDZ1/2 PSD-95-PAGFP together with untagged WT PSD-95 (G) or expressing untagged $\triangle$ PDZ1/2 PSD-95 together with WT PSD-95-PAGFP $(\boldsymbol{H})$. Neurons were transfected with $\triangle$ PDZ1/2 and WT forms of PSD-95 in a ratio of 3:1 to favor expression of the $\triangle$ PDZ1/2 form. I, Fluorescence after photoactivation for the following combinations of PSD-95 constructs: WT-PAGFP $+\Delta$ PDZ1/ 2-untagged ( $n=31 / 4$ spines/cells) or $\Delta$ PDZ1/2-PAGFP + WT-untagged ( $n=38 / 7$ spines/cells). J, Average time courses of fluorescence for PSD-95-PAGFP + mRFP control ( $n=36 / 4$ spines/neurons) and PSD-95-PAGFP + shNR1 $(n=32 / 5$ spines/ neurons). To allow for efficient knock-down of endogenous NR1, neurons were transfected $10 \mathrm{~d}$ before imaging. we examined a series of deletion and mutant constructs of PSD-95 that were tagged with PAGFP (Craven et al., 1999; Schnell et al., 2002). Our goal was both to identify domains that are necessary to form a stable lattice in the PSD as well as to identify those that are necessary for incorporation into a previously existing lattice (Fig. 2). A palmitoylation-deficient mutant of PAGFP-tagged PSD-95 (C3,5S PSD-95-PAGFP) was highly unstable in individual spines compared with WT PSD-95 (Fig. $2 A$ ). Indeed, $\sim 80 \%$ of C3,5S PSD-95 fluorescence was lost within 5 min of photoactivation (vs $\sim 7 \%$ for WT PSD-95; $p<0.05$ ) (Fig. $2 B-D$ ). A second photoactivation pulse returned $\mathrm{C} 3,5 \mathrm{~S}$ PSD-95-PAGFP fluorescence to its initial level, confirming that the rapid loss of fluorescence was due to exchange of photoactivated for unactivated PAGFP-tagged proteins in the spine. The lack of stability of C3,5S PSD-95-PAGFP did not reflect a dominant-negative phenotype that disrupted stabilization of the entire PSD, as expression of untagged C3,5S mutant PSD-95 did not perturb the normal stability of PAGFP-tagged, WT PSD-95 (Fig. $2 E$ ). Thus, palmitoylation of PSD-95 is necessary for its incorporation into a stable lattice of PSD-95 molecules.

\section{Protein-protein interactions necessary} for PSD-95 stability

Following its lipid-anchored $\mathrm{N}$ terminus, PSD-95 contains a pair of PSD-95/Disc large (Dlg)/zona occludens-1 (ZO-1) (PDZ) domains which bind to PDZ-binding motifs in ion channels such as NMDA receptors and Shaker family $\mathrm{K}$ channels, in members of the TARP family, as well as in numerous other signaling and scaffolding proteins (for review, see Kim and Sheng, 2004). A third PDZ domain, a Src homology (SH3) domain and a catalytically inactive guanylate kinase (GK) domain provide additional protein interaction sites spanning the remainder of the primary structure of PSD-95. For convention, we refer to the initial portion of PSD-95 including PDZ domains 1 and 2 as N-terminal and the remainder of PSD-95 including the third PDZ domain, $\mathrm{SH} 3$ and GK domains as C-terminal. Removing the third PDZ, SH3 and GK domains of PSD-95 ( $\Delta$ PDZ3/SH3/GK) or the $\mathrm{SH} 3$ and GK domains alone $(\Delta \mathrm{SH} 3 /$ GK) did not alter the stability of PSD-95 within its $\mathrm{N}$ terminus and that the initial 13 aa within PSD95 are both necessary and sufficient for multimerization (Craven et al., 1999; El-Husseini et al., 2000b; Christopherson et al., 2003).

To understand which domains control the rate of PSD-95 exchange in the PSD and thereby might be targets for regulation, compared with WT PSD-95 (Figs. $3 B, C, E, 4 B, E$ ). In contrast, deletion of the first $2 \mathrm{PDZ}$ domains $(\triangle \mathrm{PDZ} 1 / 2)$ significantly increased the rate of PSD-95 turnover to a level intermediate to that of C3,5S and WT PSD-95 (Fig. 3D,F) $(p<0.05)$. This intermediate stability could reflect either a general destabilization of the 
PSD or a molecule-autonomous failure of $\triangle \mathrm{PDZ} 1 / 2$ PSD-95 to be fully anchored in the PSD. To distinguish these possibilities, we assessed whether the expression of $\Delta$ PDZ1/2 PSD-95 interfered with the stability of WT PSD-95. $\triangle$ PDZ1/2 and WT PSD-95 were coexpressed in a 3:1 ratio to bias expression levels toward an abundance of the mutant form with, in separate experiments, the PAGFP tag present on either the WT or mutant protein. Comparison of these two conditions revealed that WT PSD-95-PAGFP remained more stable than $\triangle \mathrm{PDZ} 1 / 2$ PSD-95PAGFP (Fig. 3G- $I$ ), suggesting that incorporation of $\Delta \mathrm{PDZ} 1 / 2 \mathrm{PSD}-95$ within the PSD does not undermine the stability of WT PSD-95.

Since the sites of interaction with NMDAR subunits NR1 and NR2 are within the first two PDZ domains of PSD-95 (Kornau et al., 1995; Washbourne et al., 2002), and NMDARs are thought to play a role in synapse stabilization (Alvarez et al., 2007), we examined if NMDARs are necessary for the stabilization of PSD-95. In neurons in which NR1 was knocked down by RNA interference, conditions that eliminate synaptic NMDAR currents (Alvarez et al., 2007), PSD-95PAGFP stability was similar to wild-type (Fig. 3J). Thus, although the stability of PSD-95 requires $\mathrm{PDZ1/2}$, interactions with NMDA receptors are not strictly required. Rather, multiple protein partners within the PSD may interact with PDZ1/2 to promote PSD-95 stability.

Similar analysis was performed to determine the requirement of C-terminal domains of PSD-95 for its stability in the PSD (Fig. 4). Via its SH3 and GK domains, the C terminus of PSD-95 engages scaffolding proteins such as GKAP and AKAP79/150, which form a second tier of structural interactions within the PSD and maintain signaling complexes involved in the reorganization of the actin cytoskeleton and the initiation of signal transduction cascades (Kim and Sheng, 2004). We found that deletion of the SH3 and GK domains had no effect on stability of PSD-95 in the spine (Fig. $4 A, B, E$ ). This indicates that, in the context of endogenous PSD-95 expression, N-terminal interactions are necessary and sufficient for retention within a stabilized PSD-95 lattice. However, recent experiments using a molecular replacement strategy in which short-hairpin RNA (shRNA) sequences are used to knock-down endogenous PSD-95 and RNA interferenceresistant exogenous PSD-95 is expressed have demonstrated that the SH3 and GK domains are necessary to maintain synaptic strength (Xu et al., 2008). We adopted a similar approach to determine if endogenous PSD-95 supplies interactions via its intact $\mathrm{SH} 3$ and GK domains sufficient to stabilize exogenous PSD-95 lacking these domains. Under replacement conditions, deletion of both the $\mathrm{SH} 3$ and GK domains significantly increased PSD-95-PAGFP turnover compared with deletion of the GK domain alone (Fig. 4C-E). Therefore, the $\mathrm{SH} 3$ domain provides interactions that are necessary for the establishment of a stable pool of PSD-95 within the PSD, but which are dispensable for incorporation into a preexisting lattice.

\section{Rapid loss of PSD-95 from dendritic spines induced by NMDAR activation}

Many forms of stimulus-evoked synaptic plasticity are mediated by the insertion or removal of proteins from the PSD following activation of NMDARs (Malinow and Malenka, 2002; Kim and Sheng, 2004; Ward et al., 2004; Foukas et al., 2006). We examined whether the stability of PSD-95 was affected by activation of NMDARs by briefly stimulating hippocampal slice cultures with NMDA, an established protocol for the chemical induction of LTD (Kameyama et al., 1998; Lee et al., 1998). NMDAR activation provoked the rapid removal of $\sim 60 \%$ of previously stable PSD-95-PAGFP from spine heads (Fig. 5A, B,E). This loss of signal might arise from either a transient disruption of the stability of PSD-95 and exchange of photoactivated for unactivated PSD95-PAGFP or from a decrease in the size of the stable pool of PSD-95 within the spine head. Delivery of a second photoactivating pulse failed to restore green fluorescence, indicating a decrease in the number of tethered PSD-95-PAGFP molecules in the spine (Fig. $5 B, E$, PA', $p<0.05$ ). Moreover, photoactivation of an independent set of spines $30 \mathrm{~min}$ after NMDA stimulation yielded fluorescence levels that were significantly lower compared with those reached in spines activated before the induction protocol (supplemental Fig. $3 A-D$, available at www.jneurosci.org as supplemental material) $(p<0.05)$. The NMDA-induced de- 


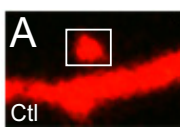

Before PA
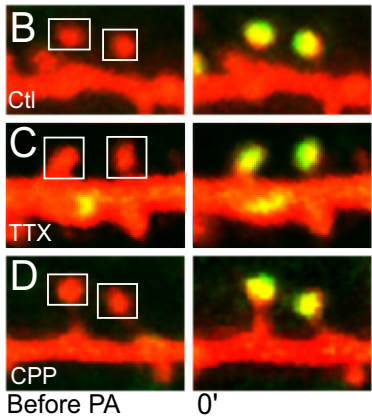

Before PA
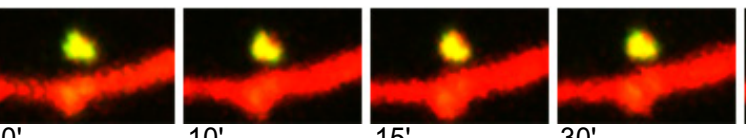

$10^{\prime}$

15

$30^{\prime}$
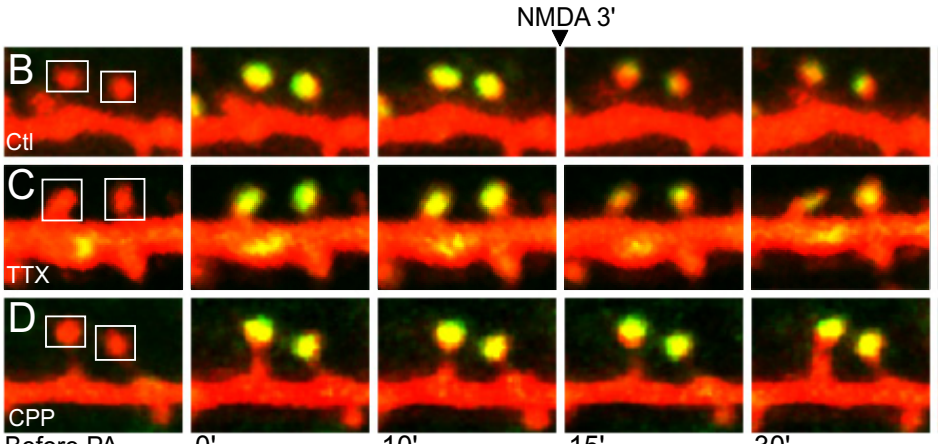

$\mathrm{E}$

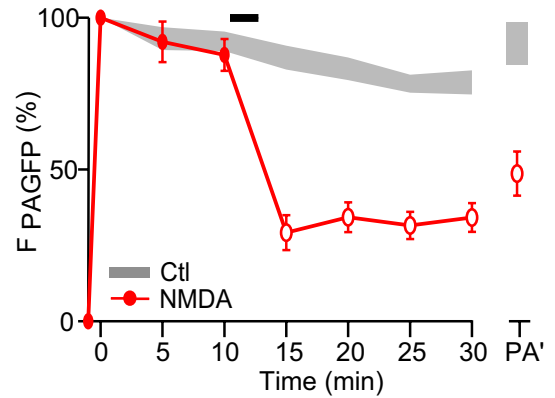

$\mathrm{F}$

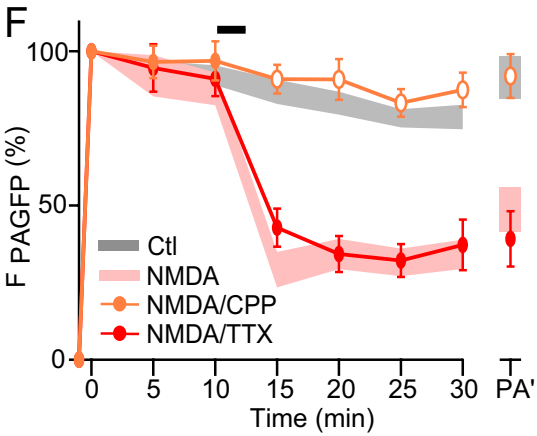

G

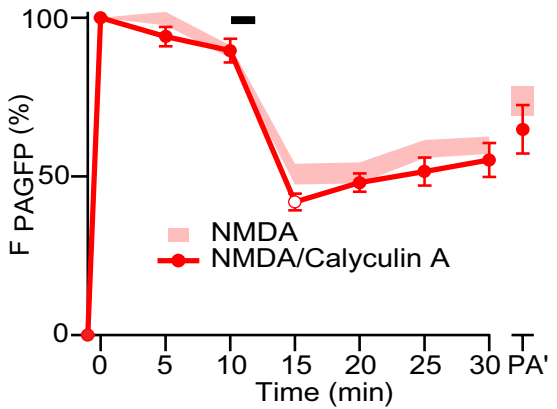

$\mathrm{H}$

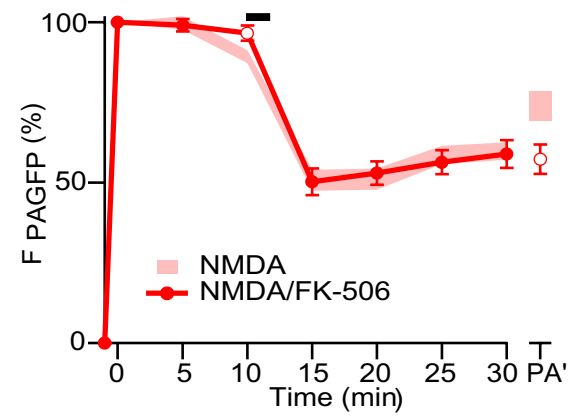

Figure 5. Activation of NMDA receptors triggers the rapid removal of PAGFP-tagged PSD-95 from dendritic spines. $A$, Representative time lapse imaging of a dendritic spine from a neuron expressing dsRed and PSD-95-PAGFP (Ctl) following photoactivation. $\boldsymbol{B}$, As in $\boldsymbol{A}$ for spines that were exposed to $20 \mu \mathrm{m}$ NMDA for 3 min starting after collection of the $t=10$ min image. $C, D, A s$ in $\boldsymbol{B}$ for spines in slices that were incubated in either $1 \mu \mathrm{m}$ TTX (C) or $20 \mu \mathrm{M}$ (PP $(\boldsymbol{D})$. $\boldsymbol{E}$, Average time courses of PSD-95-PAGFP fluorescence in spines of slices stimulated with NMDA (red circles, $n=43 / 17$ spines/cells). Open markers indicate $p<0.05$ compared to results from spines of unstimulated control neurons (shaded gray region). Black bar indicates NMDA application (20 $\mu \mathrm{M}, 3 \mathrm{~min}) . \boldsymbol{F}$, Average time courses of PSD-95-PAGFP fluorescence in spines of slices stimulated with NMDA in the presence of either TTX (red circles) or CPP (orange circles) ( $n=35 / 7$ and $n=21 / 6$ spines/cells, respectively). Open markers indicate $p<0.05$ compared to results from NMDA-stimulated neurons in control conditions (pink shaded region, replotted from $\boldsymbol{E}$ ). The shaded gray region shows behavior of PSD-95-PAGFP in unstimulated neurons for comparison. $\boldsymbol{G}, \boldsymbol{H}$, Effect of the phosphatase inhibitors Calyculin A (G, red circles, $n=33 / 4$ spines/cells) and FK-506 ( $\boldsymbol{H}$, red circles, $n=29 / 5$ spines/cells) on average time courses of PSD-95-PAGFP fluorescence in NMDA-stimulated spines. Black bar indicates NMDA application ( $40 \mathrm{~mm}, 3 \mathrm{~min}$ ). These results are plotted along with an independent, interleaved set of control NMDA-treated spines (pink shaded region, open markers indicate $p<0.05$ compared to control) ( $n=42 / 8$ spines/cells).

crease in the size of the tethered pool of PSD-95-PAGFP was mediated by NMDAR activation in the imaged cell as it was unaffected by the voltage-sensitive Na channel antagonist TTX but blocked by the NMDAR antagonist CPP (Fig. 5C,D,F). The chemical LTD protocol also induced a slight but transient decrease in the size of the spine head (supplemental Fig. $3 E-G$, available at www.jneurosci.org as supplemental material). Since activities of PP1 and PP2B are required for expression of

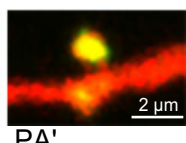

PA

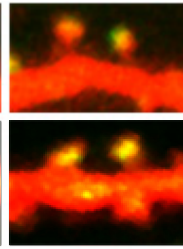

NMDAR-mediated LTD (Mulkey et al., 1993, 1994), we examined the requirements of these phosphatases in NMDAstimulated loss of PSD-95 from spines. We found that neither treatment with the PP1/PP2A inhibitor Calyculin A nor the PP2B inhibitor FK-506 affected this process (Fig. 5G,H).

\section{PDZ domains 1 and 2 mediate the NMDA-dependent regulation of PSD-95 stability}

To determine what protein domains of PSD-95 are necessary for the NMDAevoked removal of PSD-95 from the spine, we examined the effect of NMDAR activation on the stability of PSD-95 deletion constructs. The behavior of $\triangle \mathrm{PDZ} /$ SH3/GK PSD-95-PAGFP was similar to that of WT PSD-95-PAGFP indicating that these domains are not required for NMDA-evoked removal of PSD-95 (Fig. $6 A, C)$. In contrast, NMDA had no effect on the stability of $\triangle \mathrm{PDZ} 1 / 2$ PSD-95PAGFP, demonstrating that the $\mathrm{N}$ terminus of PSD-95 including PDZ domains 1 and 2 is necessary and sufficient for NMDAR-dependent regulation of PSD-95 stability (Fig. 6B,D).

\section{Discussion}

In this study, we tagged PSD-95 and other PSD proteins with photoactivatable GFP and examined their stabilities within dendritic spines. We find that compared with other proteins such as GluR2, Stargazin, CaMKII $\alpha$, and CaMKII $\beta$, PSD-95 is remarkably stable, turning over very little over the course of $30 \mathrm{~min}$. Palmitoylation of PSD-95 is critical for its incorporation into stable structures within the spine head and the N-terminal PDZ domains 1 and 2 further stabilize PSD-95. While more C-terminal domains are unnecessary for the stabilization of PSD-95 within a preexisting PSD, the C-terminal $\mathrm{SH} 3$ domain is required for initial stabilization of this structure. Using a chemical model of NMDAR-dependent LTD, we find that PSD-95 is rapidly destabilized by NMDAR activation, an effect mediated by PDZ domains 1 and 2. Our results establish that both lipid anchoring and protein-protein interactions stabilize PSD-95 within the PSD and that PSD-95 stability and numbers are regulated during the chemical induction of LTD.

\section{Organization of a lattice-like structure by PSD-95}

Multiple lines of evidence suggest that PSD-95 helps form, organize, and stabilize PSD structure. As would be expected for a key structural component of the PSD, PSD-95 is abundant with an estimated 300 copies present at an average 
PSD (Chen et al., 2005; Sugiyama et al., 2005). PSD-95 can self-associate: its $\mathrm{N}$-terminal palmitoylation domain mediates multimerization and also targets PSD-95 to the plasma membrane (Christopherson et al., 2003). Here, we find that PSD-95 is highly stable within the PSD (Fig. $1 A, D-E$ ). In contrast, CaMKII $\alpha$ and CaMKII $\beta$, while even more abundant, are significantly less stable (Fig. 1C-E) (see also Lee et al., 2009).

The localization and arrangement of PSD-95 molecules within the PSD are consistent with a role in forming a highly ordered structure into which other PSD proteins are integrated and stabilized. Within the laminar structure of the PSD, PSD-95 is localized nearest the inner leaflet of the plasma membrane (Valtschanoff and Weinberg, 2001). Reconstructions of PSDs using EM tomography revealed an ordered, lattice-like array of vertical filaments which were immunopositive for PSD-95 (Chen et al., 2008). Transmembrane structures whose dimensions are consistent with AMPA and NMDA receptors were associated with these filaments as well as horizontal linkages whose identities might correspond to scaffolding proteins such as GKAP/SAPAP which bind the C-terminal portion of PSD-95.

Taking advantage of the fact that PSD-95 lacking SH3 and GK domains is fully competent to enter the PSD and be stabilized (Fig. $4 B, E$ ), we investigated whether these domains might instead be required in the overall formation of PSD structure within which individual PAGFP-tagged PSD-95 molecules are integrated. We find that in the absence of endogenous PSD-95, exogenous PSD-95 lacking both the SH3 and GK domains is less stable than a mutant lacking the GK domain alone (Fig. $4 C, D, F$ ). This result suggests that the $\mathrm{SH} 3$ domain of PSD-95 mediates interactions with proteins important for the formation of a stable PSD. The protein(s) that interact with the $\mathrm{SH} 3$ domain to perform this function remain unknown but may include other MAGUK family proteins.

\section{Role of PSD-95 destabilization in the expression of LTD}

In addition to a general role in maintaining and organizing PSD structure, PSD-95 plays a specific role in the induction of LTD. PSD-95 is anchored by its N terminus to NMDA receptors which generate calcium signals during LTD and by its $\mathrm{C}$ terminus to calcineurin via AKAP79/150 (Coghlan et al., 1995; Kornau et al., 1995; Klauck et al., 1996; Bhattacharyya et al., 2009). Therefore, PSD-95 may localize signaling components needed for LTD induction in proximity to NMDARs. Indeed, not only has PSD-95 been shown to be required for the expression of LTD but its role in LTD induction has been dissociated from its direct role in anchoring TARP/AMPA receptor complexes at the synapse and thereby regulating synaptic strength (Xu et al., 2008). Furthermore, a recent study established that the C-terminal SH3 and GK domains of PSD-95 are required for NMDAR-dependent endocytosis of AMPARs and that this effect is likely mediated by activation of AKAP79/150-tethered calcineurin (Bhattacharyya et al., 2009).

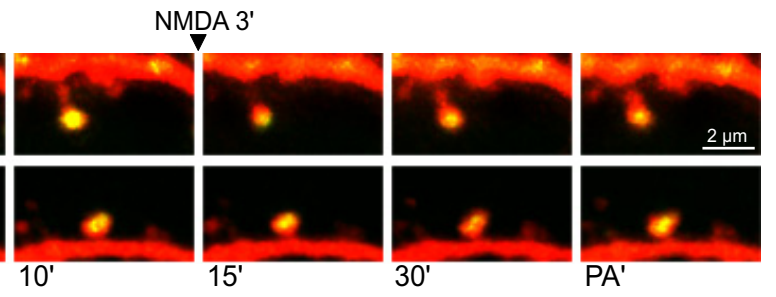

$\mathrm{D}$

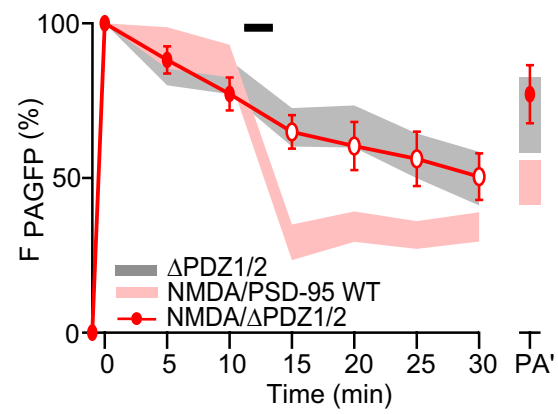

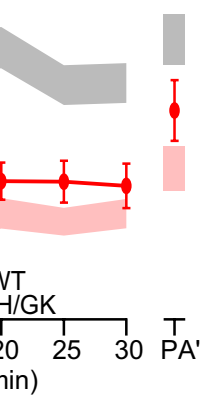

Figure 6. N-terminal domains including PDZ1/2 are necessary and sufficient for the NMDA-induced removal of PAGFP-tagged .

NMDAR-dependent LTD is expressed via a reduction in synaptic AMPARs and is accompanied by reductions in spine size (Oliet et al., 1996; Carroll et al., 1999; Bredt and Nicoll, 2003; Zhou et al., 2004). Given the tight correlation between synaptic strength, spine size, and PSD size, it would be predicted that LTD expression would involve partial disassembly of the PSD. To address whether the number and stability of synaptic PSD-95 proteins are regulated during LTD, we chemically stimulated NMDARs using a protocol that induces long-term depression (chemLTD) at synapses throughout the slice (Kameyama et al., 1998; Lee et al., 1998). We found that brief application of NMDA rapidly removes $\sim 60 \%$ of PSD-95-PAGFP from the spine head (Fig. 5), reflecting both a rapid release of tethered photoactivated proteins and a persistent decrease in the number of tethered PSD-95 molecules in the spine head. NMDA-induced loss of PSD-95 from the spine required the same protein domains necessary for basal stability of PSD-95; thus, deletion of the first 2 PDZ domains but not the third PDZ, SH3 or GK domains prevented NMDA-induced loss of PSD-95. Thus, rapid destabilization of spine-localized PSD-95 is responsible for the loss of PSD-95 levels seen following chemLTD (Smith et al., 2006; Bhattacharyya et al., 2009). Furthermore, our studies reveal that LTD induction is accompanied by a loss of a fraction of the previously anchored PSD-95 but not by full exchange of all the PSD-95 molecules in the spine.

Recent studies have identified two different modes of regulation that might contribute to the destabilization of PSD-95 during LTD. During LTP induction, phosphorylation of serine 73 moderates spine growth by destabilizing PSD-95 within the PSD and transiently decreasing PSD-95 levels within the spine (Steiner et al., 2008). As serine 73 is positioned within PDZ domains 1 and 2, a role for phosphorylation of this site in PSD-95 destabilization is consistent with our molecular analysis. In addition, phosphorylation at serine 295 has been shown to positively correlate with 
synaptic PSD-95 content (Kim et al., 2007). Dephosphorylation of this site was shown to occur following NMDA stimulation, consistent with a role in LTD.

Activation of protein phosphatases is required for the expression of LTD, with NMDAR-mediated activation of calcineurin (PP2B) leading to further activation of downstream phosphatases such as PP1 (Mulkey et al., 1993, 1994). Yet a growing body of evidence points toward a dissociation between the mechanisms responsible for different forms and aspects of LTD (Kameyama et al., 1998; Zhou et al., 2004; Morishita et al., 2005; Wang et al., 2007). While depression of synaptic responses requires activation of both calcineurin and PP1, spine shrinkage accompanying LTD requires activation of calcineurin and not PP1 (Zhou et al., 2004; Wang et al., 2007). Similarly, chemLTD-evoked internalization of AMPA receptors requires calcineurin but not PP1 and/or PP2A (Beattie et al., 2000) (but see also Kameyama et al., 1998). We found that neither inhibition of PP1 and PP2A nor inhibition of calcineurin affected NMDA-induced removal PSD-95, consistent with mechanistic dissociations between depression of synaptic currents, spine shrinkage, and rapid disassembly of the PSD.

\section{Conclusion}

The core set of protein interactions necessary to form and stabilize the lattice-like structure of the PSD is poorly understood. Here, we confirm that PSD-95 is such a core protein by demonstrating that PSD-95 is highly stable within the PSD, even more so than other representative synaptic proteins. Rather than contributing additively, we show that distinct interaction domains stabilize PSD-95 by different mechanisms. N-terminal palmitoylation facilitates the entry of PSD-95 into the postsynaptic lattice while PDZ domains 1 and 2 stabilize PSD-95 after it has entered the lattice. Within an established PSD, C-terminal domains do not contribute to the stabilization of PSD-95, consistent with a role in anchoring other, less stable molecules. Yet in the absence of endogenous PSD-95, the C-terminal SH3 domain becomes necessary for stability. This finding suggests that multivalent $\mathrm{N}$-terminal interactions may bridge PSD-95 molecules, enforcing overall PSD structure, and maintaining the stability of truncated PSD-95 in the presence of endogenous, WT protein. Furthermore, these same N-terminal interactions are necessary for rapid, NMDAR-dependent trafficking of PSD-95 out of the spine. Thus, by determining the stability of itself and other proteins within the PSD, PSD-95 plays a central role in maintaining synaptic function and gating structural and functional changes necessary for synaptic plasticity.

\section{References}

Alvarez VA, Ridenour DA, Sabatini BL (2007) Distinct structural and ionotropic roles of NMDA receptors in controlling spine and synapse stability. J Neurosci 27:7365-7376.

Beattie EC, Carroll RC, Yu X, Morishita W, Yasuda H, von Zastrow M, Malenka RC (2000) Regulation of AMPA receptor endocytosis by a signaling mechanism shared with LTD. Nat Neurosci 3:1291-1300.

Bellone C, Nicoll RA (2007) Rapid bidirectional switching of synaptic NMDA receptors. Neuron 55:779-785.

Bhattacharyya S, Biou V, Xu W, Schlüter O, Malenka RC (2009) A critical role for PSD-95/AKAP interactions in endocytosis of synaptic AMPA receptors. Nat Neurosci 12:172-181.

Blanpied TA, Kerr JM, Ehlers MD (2008) Structural plasticity with preserved topology in the postsynaptic protein network. Proc Natl Acad Sci U S A 105:12587-12592.

Bloodgood BL, Sabatini BL (2005) Neuronal activity regulates diffusion across the neck of dendritic spines. Science 310:866-869.

Bredt DS, Nicoll RA (2003) AMPA Receptor Trafficking at Excitatory Synapses. Neuron 40:361-379.
Carroll RC, Lissin DV, von Zastrow M, Nicoll RA, Malenka RC (1999) Rapid redistribution of glutamate receptors contributes to long-term depression in hippocampal cultures. Nat Neuroscience 2:454.

Chen X, Vinade L, Leapman RD, Petersen JD, Nakagawa T, Phillips TM, Sheng M, Reese TS (2005) Mass of the postsynaptic density and enumeration of three key molecules. Proc Natl Acad Sci U S A 102:1155111556.

Chen X, Winters C, Azzam R, Li X, Galbraith JA, Leapman RD, Reese TS (2008) Organization of the core structure of the postsynaptic density. Proc Natl Acad Sci U S A 105:4453-4458.

Choe CU, Ehrlich BE (2006) The Inositol 1,4,5-Trisphosphate Receptor (IP3R) and Its Regulators: Sometimes Good and Sometimes Bad Teamwork. Sci STKE 2006:re15.

Christopherson KS, Sweeney NT, Craven SE, Kang R, El-Husseini Ael-D, Bredt DS (2003) Lipid- and protein-mediated multimerization of PSD95: implications for receptor clustering and assembly of synaptic protein networks. J Cell Sci 116:3213-3219.

Coghlan VM, Perrino BA, Howard M, Langeberg LK, Hicks JB, Gallatin WM, Scott JD (1995) Association of protein kinase A and protein phosphatase 2B with a common anchoring protein. Science 267:108-111.

Craven SE, El-Husseini AE, Bredt DS (1999) Synaptic Targeting of the Postsynaptic Density Protein PSD-95 Mediated by Lipid and Protein Motifs. Neuron 22:497-509.

Ehlers MD (2003) Activity level controls postsynaptic composition and signaling via the ubiquitin-proteasome system. Nat Neurosci 6:231-242.

El-Husseini AE, Schnell E, Chetkovich DM, Nicoll RA, Bredt DS (2000a) PSD-95 involvement in maturation of excitatory synapses. Science 290:1364-1368.

El-Husseini AE, Craven SE, Chetkovich DM, Firestein BL, Schnell E, Aoki C, Bredt DS (2000b) Dual palmitoylation of PSD-95 mediates its vesiculotubular sorting, postsynaptic targeting, and ion channel clustering. J Cell Biol 148:159-172.

Foukas LC, Claret M, Pearce W, Okkenhaug K, Meek S, Peskett E, Sancho S, Smith AJH, Withers DJ, Vanhaesebroeck B (2006) Critical role for the p110[alpha] phosphoinositide-3-OH kinase in growth and metabolic regulation. Nature 441:366-370.

Gray NW, Weimer RM, Bureau I, Svoboda K (2006) Rapid redistribution of synaptic PSD-95 in the neocortex in vivo. PLoS Biology 4:e370.

Inoue A, Okabe S (2003) The dynamic organization of postsynaptic proteins: translocating molecules regulate synaptic function. Curr Opin Neurobiol 13:332-340.

Kameyama K, Lee HK, Bear MF, Huganir RL (1998) Involvement of a postsynaptic protein kinase A substrate in the expression of homosynaptic long-term depression. Neuron 21:1163-1175.

Kelsch W, Lin CW, Lois C (2008) Sequential development of synapses in dendritic domains during adult neurogenesis. Proc Natl Acad Sci U S A 105:16803-16808.

Kennedy MB (2000) Signal-processing machines at the postsynaptic density. Science 290:750-754.

Kim E, Sheng M (2004) PDZ domain proteins of synapses. Nat Rev Neurosci 5:771-781.

Kim MJ, Futai K, Jo J, Hayashi Y, Cho K, Sheng M (2007) Synaptic Accumulation of PSD-95 and Synaptic Function Regulated by Phosphorylation of Serine-295 of PSD-95. Neuron 56:488-502.

Klauck TM, Faux MC, Labudda K, Langeberg LK, Jaken S, Scott JD (1996) Coordination of three signaling enzymes by AKAP79, a mammalian scaffold protein. Science 271:1589-1592.

Koh YH, Popova E, Thomas U, Griffith LC, Budnik V (1999) Regulation of DLG localization at synapses by CaMKII-dependent phosphorylation. Cell 98:353-363.

Kornau HC, Schenker LT, Kennedy MB, Seeburg PH (1995) Domain interaction between NMDA receptor subunits and the postsynaptic density protein PSD-95. Science 269:1737-1740.

Kuriu T, Inoue A, Bito H, Sobue K, Okabe S (2006) Differential control of postsynaptic density scaffolds via actin-dependent and -independent mechanisms. J Neurosci 26:7693-7706.

Lee HK, Kameyama K, Huganir RL, Bear MF (1998) NMDA induces longterm synaptic depression and dephosphorylation of the GluR1 subunit of AMPA receptors in hippocampus. Neuron 21:1151-1162.

Lee SJ, Escobedo-Lozoya Y, Szatmari EM, Yasuda R (2009) Activation of CaMKII in single dendritic spines during long-term potentiation. Nature 458:299-304. 
Malinow R, Malenka RC (2002) AMPA receptor trafficking and synaptic plasticity. Annu Rev Neurosci 25:103-126.

Morishita W, Marie H, Malenka RC (2005) Distinct triggering and expression mechanisms underlie LTD of AMPA and NMDA synaptic responses 8:1043-1050.

Mulkey RM, Herron CE, Malenka RC (1993) An essential role for protein phosphatases in hippocampal long-term depression. Science 261:10511055.

Mulkey RM, Endo S, Shenolikar S, Malenka RC (1994) Involvement of a calcineurin/inhibitor-1 phosphatase cascade in hippocampal long-term depression. Nature 369:486-488.

Okabe S, Urushido T, Konno D, Okado H, Sobue K (2001) Rapid Redistribution of the Postsynaptic Density Protein PSD-Zip45 (Homer 1c) and Its Differential Regulation by NMDA Receptors and Calcium Channels. J Neurosci 21:9561-9571.

Oliet SH, Malenka RC, Nicoll RA (1996) Bidirectional control of quantal size by synaptic activity in the hippocampus. Science 271:1294-1297.

Otmakhov N, Tao-Cheng JH, Carpenter S, Asrican B, Dosemeci A, Reese TS, Lisman J (2004) Persistent accumulation of calcium/calmodulindependent protein kinase II in dendritic spines after induction of NMDA receptor-dependent chemical long-term potentiation. J Neurosci 24:93249331.

Patterson GH, Lippincott-Schwartz J (2002) A photoactivatable GFP for selective photolabeling of proteins and cells. Science 297:1873-1877.

Patterson GH, Lippincott-Schwartz J (2004) Selective photolabeling of proteins using photoactivatable GFP. Methods 32:445-450.

Petersen JD, Chen X, Vinade L, Dosemeci A, Lisman JE, Reese TS (2003) Distribution of postsynaptic density (PSD)- 95 and $\mathrm{Ca}^{2+} /$ calmodulindependent protein kinase II at the PSD. J Neurosci 23:11270-11278.

Scannevin RH, Huganir RL (2000) Postsynaptic organization and regulation of excitatory synapses. Nat Rev Neurosci 1:133-141.

Schnell E, Sizemore M, Karimzadegan S, Chen L, Bredt DS, Nicoll RA (2002) Direct interactions between PSD-95 and stargazin control synaptic AMPA receptor number. Proc Natl Acad Sci U S A 99:13902-13907.

Sharma K, Fong DK, Craig AM (2006) Postsynaptic protein mobility in dendritic spines: Long-term regulation by synaptic NMDA receptor activation. Molecular and Cellular Neuroscience 31:702-712.

Shen K, Meyer T (1999) Dynamic control of CaMKII translocation and localization in hippocampal neurons by NMDA receptor stimulation. Science 284:162-166.

Sheng M (2001) Molecular organization of the postsynaptic specialization. Proc Natl Acad Sci U S A 98:7058-7061.
Sheng M, Hoogenraad CC (2007) The postsynaptic architecture of excitatory synapses: a more quantitative view. Annu Rev Biochem 76:823-847.

Smith KE, Gibson ES, Dell'Acqua ML (2006) cAMP-dependent protein kinase postsynaptic localization regulated by NMDA receptor activation through translocation of an A-kinase anchoring protein scaffold protein. J Neurosci 26:2391-2402.

Stein V, House DR, Bredt DS, Nicoll RA (2003) Postsynaptic density-95 mimics and occludes hippocampal long-term potentiation and enhances long-term depression. J Neurosci 23:5503-5506.

Steiner P, Higley MJ, Xu W, Czervionke BL, Malenka RC, Sabatini BL (2008) Destabilization of the postsynaptic density by PSD-95 serine 73 phosphorylation inhibits spine growth and synaptic plasticity. Neuron 60:788-802.

Stoppini L, Buchs PA, Muller D (1991) A simple method for organotypic cultures of nervous tissue. J Neurosci Methods 37:173-182.

Sugiyama Y, Kawabata I, Sobue K, Okabe S (2005) Determination of absolute protein numbers in single synapses by a GFP-based calibration technique. Nat Methods 2:677-684.

Tsuriel S, Geva R, Zamorano P, Dresbach T, Boeckers T, Gundelfinger ED, Garner CC, Ziv NE (2006) Local sharing as a predominant determinant of synaptic matrix molecular dynamics. PLoS Biol 4:e271.

Valtschanoff JG, Weinberg RJ (2001) Laminar Organization of the NMDA Receptor Complex within the Postsynaptic Density. J Neurosci 21:12111217.

Wang XB, Yang Y, Zhou Q (2007) Independent expression of synaptic and morphological plasticity associated with long-term depression. J Neurosci 27:12419-12429.

Ward ME, Wu JY, Rao Y (2004) From the Cover: Visualization of spatially and temporally regulated N-WASP activity during cytoskeletal reorganization in living cells. Proc Natl Acad Sci U S A 101:970-974.

Washbourne P, Bennett JE, McAllister AK (2002) Rapid recruitment of NMDA receptor transport packets to nascent synapses. Nat Neurosci 5:751-759.

Xu W, Schlüter OM, Steiner P, Czervionke BL, Sabatini B, Malenka RC (2008) Molecular dissociation of the role of PSD-95 in regulating synaptic strength and LTD. Neuron 57:248-262.

Yoshii A, Constantine-Paton M (2007) BDNF induces transport of PSD-95 to dendrites through PI3K-AKT signaling after NMDA receptor activation. Nat Neurosci 10:702-711.

Zhou Q, Homma KJ, Poo MM (2004) Shrinkage of dendritic spines associated with long-term depression of hippocampal synapses. Neuron 44: $749-757$. 